\title{
Hue discrimination related to linguistic habits
}

\author{
JAMES KOPP1 AND HARLAN LANE, CENTER FOR \\ RESEARCH ON LANGUAGE AND LANGUAGE \\ BEHAVIOR ${ }^{2}$, UNIVERSITY OF MICHIGAN, Ann Arbor, Mich. \\ 48104
}

Reasoning from recent psycholinguistic research, it was predicted that discrimination between two monochromatic stimuli would be poorer when they belong to the same color category, better when they belong to different color categories in the language of the observer. Accordingly, it was predicted that hue discrimination functions vary systematically from one language community to the next to the extent that the different languages categorize the spectrum differently. Both predictions were supported by color labeling and discrimination measures obtained from speakers of English and of Tzotzil.

Recent research in psycholinguistics (Lane, 1966, 1967) indicates that whenever a stimulus continuum is effectively partitioned into perceptual categories there are several behavioral consequences including: (a) the probability of assigning a stimulus to a given category tends toward zero or unity-that is, the distributions of identification probabilities over the continuum are step-like, with broad plateaus and sharp transitions; (b) the mean latency of identifying the stimuli is short over the plateaus, within categories, and long at the transition points, at category boundaries; (c) the accuracy of discriminating between two stimuli is poor if they both belong to the same category, better if they belong to different categories-that is, there is discrimination enhancement at category boundaries.

The effective partitioning of the stimulus continuum may be accomplished by the language community (Lane, 1965) presumably over years of verbal conditioning, or by the experimenter in the laboratory, working more rapidly and systematically (Cross \& Lane, 1962; Lane \& Moore, 1962; Cross, Lane, \& Sheppard, 1965).

It has long been recognized that language habits similarly partition the color continuum (Ray, 1953). From what has been said above, we would expect that the relative probability of color names would be distributed stepwise as a function of wavelength and that their latencies would be short within color classes and long at class boundaries-both confirmed by Beare (1963). More interestingly, we would expect that the accuracy of discriminating two wavelengths would be poor within color categories and better across categories. Lane (1967) confirmed this expectation by superimposing the hue discrimination data of Laurens \& Hamilton (1923) on the hue labeling data of Beare.

This result is striking because it suggests the possibility that hue discrimination may not depend exclusively on the inborn sensory mechanism of the observer but also on his linguistic habits. This brazenly Whorfian line of thought leads to the prediction that hue discrimination functions vary systematically from one language community to the next, to the extent that the different languages partition the hue continuum differently.

We first confirmed the correlation of hue labeling with hue discrimination by using English-speaking college students in four 2-h sessions. We then moved the apparatus to the anthropological field station in San Cristobal de las Casas, Chiapas, Mexico where adult monolingual speakers of Tzotzil (a Mayan language) were recruited from a nearby Indian village for similar paid service. Next. we established that color categories in Tzotzil are different from those in English. Finally, we confirmed that the hue discrimination function of Tzotzil speakers is correlated with their color categories and thus differs from that of English speakers.

Method. The Tzotzil Ss were from the village of Nevencliauk near San Cristobal de las Casas. Access to these Ss was through a local informant who also served as a Spanish-Tzotzil interpreter during the actuat experiment. Two Ss, one male and one female, both monolingual in Tzotzil, participated. The male (Mariano) was 21 and the fentale (Petrona) 10 years of age.
The American English Ss (MR and AA) were female college students at the University of Michigan.

Monochromatic stimuli for identification and discrimination were generated by passing "white" ( $3200 \mathrm{deg} \mathrm{K})$ light through a continuous interference filter and thence through a $1 \mathrm{~mm}$ vertical slit. This procedure produces relatively intense spectral stimuli with a half band-width of approximately 12 $\mathrm{m} \mu$ over the entire range of visible wavelengths. Wavelength changes are produced by traversing the filter horizontally across the slit, the peak wavelength value in millimicrons being linear with the traverse distance in $\mathrm{mm}$. Stimulus intensities ranged monotonically from 62 to $86 \mathrm{~dB}$ (re: $10^{-10} \mathrm{~L}$ ) at the shortest and longest wavelengths, respectively. These values are well above the photopic threshold. Ss were seated in a completely darkened chamber, $2.5 \mathrm{ft}$ from a ground glass screen. The stimuli were projected upon the obverse side of the glass through the lens system of a standard slide projecter. The resultant stimulus image consisted of a vertical rectangle, $3 / 4 \times 5 / 16$ in. The stimulus surround was rendered completely black by masking the screen with black posterboard. All stimulus events and concomitant responses were recorded on an 8-channel event recorder. During the labeling phase, response latencies were recorded by means of a multivibrator-counter arrangement which allowed temporal resolution to within 64 msec.

In the first part of the experiment the $S$ was provided with a telegraph key and instructed to report all hue changes, however slight, in the patch of light on the screen (sweep discrimination procedure). The $\mathrm{E}$ then actuated a motor which moved the filter continuously across the slit at a slow fixed rate from right to left and back again. This yielded continually alternating ascending and descending sweeps of the spectrum from the violets (420 millimicrons) to the reds (645 millimicrons).

In the next phase, discrete wavelength values were presented tachistoscopically at $7.5 \mathrm{sec}$ intervals (stimulus duration $=.75 \mathrm{sec}$ ) according to a random protocol (hue identification procedure). The appropriate wavelengths were set manually by the $E$ by means of a dial micrometer fixed to the filter carrier. The $S$ was instructed to press one of several dimly illuminated buttons on a panel in front of him, each corresponding to one of the several most common color names extant in his language. For English speaking Ss the colors used were red, yellow, green, blue, and violet. Orange was eliminated on the basis of data published by Sternheim \& Boynton (1966). For the Indian Ss, the permissible color names were $/ \mathrm{coh} /, /$ yox/. $/ \mathrm{k}$ 'on $/$, and $/ \mathrm{ik}$ loan/. These four names were found to predominate in a color naming experiment conducted by Collier (1963) in which Tzotzil Ss assigned color names without restriction to Munsell color chips. In the Collier study, $/ \mathrm{c} \phi \mathrm{h} /, / \mathrm{k}$ 'on/, /yox/, and /ik'loan/ were associated with peak relative frequencies of $60 \%, 65 \%, 85 \%$, and $40 \%$, respectively. The peak frequencies for other names ranged from $20 \%$ to $30 \%$.

In the third phase of the experiment, pairs of adjacent stimuli from the identification phase were presented in "ABX" discrimination triads. The triads were constructed so that the third stimulus was always a replication of one of the first two, yielding four permutations per pair of stimuli: ABA. $\mathrm{ABB}, \mathrm{BAA}$, and $\mathrm{BAB}$. Each possible triad was presented 24 times according to a random protocol. The $S$ was instructed to press one of two buttons to indicate whether the third stimulus was identical to the first or the second. In this phase the stimulus duration was again $.75 \mathrm{sec}$. Stimulus onset occurred at $3 \mathrm{sec}$ intervals within triads. Triad onsets occurred at $19.5 \mathrm{sec}$ intervals.

The final phase consisted of a replication of the first phase in which the $S$ depressed a telegraph key to report the slightest hue change as the wavclength values were changed continuously over the spectrum.

Results and Discussion. Figure I (a-d) presents, for two English and two Tzotzil speakers, the obtained distributions of: identification probability, identification latency. sweep discrimination and $A B X$ discrimination. Also included are $A B X$ discrimination values which would be predicted from the labeling probabilities assuming that the Ss labeled the stimuli during the ABX phase as they did during the actual labeling task (Liberman et al, 1957).

First it will be seen that. in accord with our initial generalization. the partitioning of the continuum into perceptual categories by the language leads to poorer discrimination within categories, better discrimination across categories: this is truc for both measures of discrimination obtained from both language 


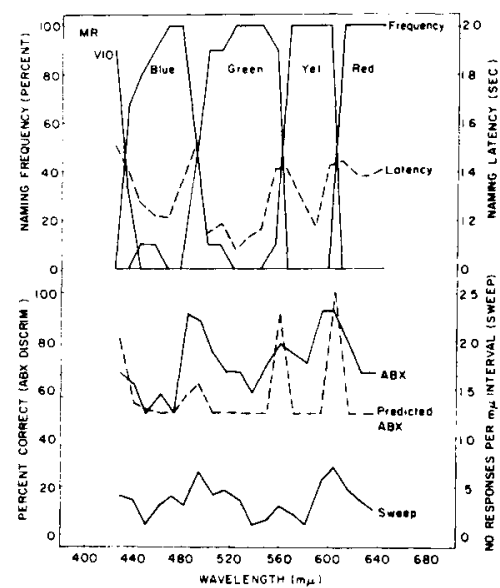

(a)

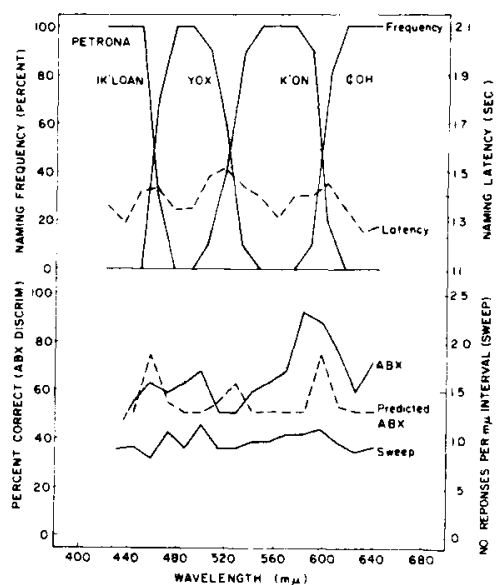

(c)

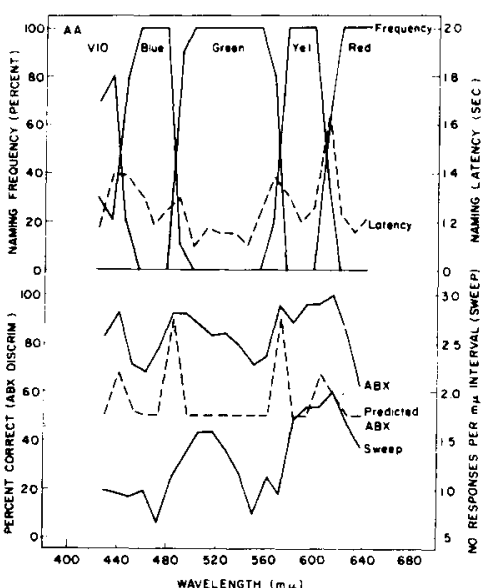

(b)

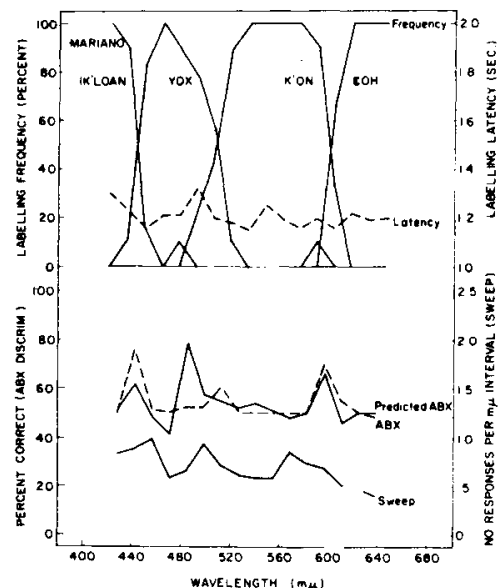

(d)
Fig. 1. Identification probability, identification latency, sweep discrimination and ABX discrimination ineasures as a function of wavelength for two English (MR and AA) and two Tzotzil (Petrona and Mariano) speakers. Each point on the functions is the mean of 10,10, 40, or 24 stimulus presentations, respectively. groups. Second, it will be noted that because of this correlation between color categories and color discrimination and because the color categories are different in the two language groups, color discrimination is different in the two language groups.

The hue labeling and hue discrimination functions obtained from English speakers are comparable to those reported by other investigators (Laurens \& Hamilton, 1923; Beare, 1963; Boynton et al, 1964; Luria, 1966). These functions differ, however, from the hue labeling and correlated hue discrimination functions obtained from the Tzotzil speakers. We are led to infer that hue discriminability functions may vary systematically from one language community to the next-to the extent that color categories in the different languages partition the hue continuum differently.

\section{REFERENCES}

BEARE, A. C. Color name as a function of wavelength. Amer. J. Psychol., $1963,76,248$

BOYNTON, R. M., SCHAFER, W., \& NEUN, M. E. Hue-wavelength relation measured by color-naming method for three retinal locations. Science, $1964,146,666$.

COLLIER, G. A. Color categories in Zinancantan. Unpublished honors thesis, Harvard College, 1963.

CROSS, D. V., \& LANE, H. L. On the discriminative control of concurrent responses: the relations among response frequency, latency, and topog raphy in auditory generalization. J. exp. Anal. Behav., 1962, 5, 487-496.

CROSS, D. V., LANE, H. L., \& SHEPPARD, W. C. Identification and discrimination functions for a visual continuum and their relation to the motor theory of speech perception. J. exp. Psychol., 1965, 70, 63-74.
LANE, H. L. The motor theory of speech perception: a critical review. Psychol. Rev., 1965, 72, 275-309.

LANE, H. L. Identification, discrimination, translation: the effects of mapping ranges of physical continua onto phoneme and sememe categories. Int. Rev. appl. Ling., 1966, 4, 215-226.

LANE, H. L. A behavioral basis for the polarity principle in linguistics. In $\mathrm{K}$. Salzinger (Ed.), Research in verbal behavior and some neurophysiological implications. New York: Academic Press, 1967. Pp. 79-98.

LANE, H. L., \& MOORE, D. J. Reconditioning a consonant discrimination in an aphasic: an experimental case history.J. speech hear. Disord., 1962, 27, 232-243.

LAURENS, H., \& HAMILTON, W. F. The sensibility of the eye to differences in wavelength. Amer. J. Physiol, 1923, 65, 547-568.

LIBERMAN, A. M., HARRIS, KATHERINE S., HOFFMAN, H. S., \& GRIFFITH, B. C. The discrimination of speech sounds within and across phoneme boundaries. J. exp. Psychol., 1957, 54, 358-368.

LURIA, S. M. Color name as a function of stimulus-intensity and duration. Amer. J. Psychol., 1967, 80, 14-27.

RAY, V. F. Human color perception and behavioral response. Trans. N. Y. Acad. Sci., 1953, 16, 98-104.

STERNHEIM, C. E., \& BOYNTON, R. M. Uniqueness of perceived hues investigated with a continuous judgmental technique. J. exp. Psychol, $1966,72,770-776$.

\section{NOTES}

1. Now at Claremont Graduate School, Claremont, California.

2. The Center for Research on Language and Language Behavior is supported in part by a contract with the Language section, U. S. Office of Education. 